\title{
Strella (film de Panos Koutras, 2009), une version queer du mythe d'CEdipe
}

Strella (A woman's way, Panos Koutras, 2009): a queer version of the CEdipus myth

\section{Sophie Coavoux}

\section{OpenEdition}

\section{Journals}

Édition électronique

URL : http://journals.openedition.org/transtexts/487

DOI : $10.4000 /$ transtexts. 487

ISSN : 2105-2549

Éditeur

Gregory B. Lee

Référence électronique

Sophie Coavoux, «Strella (film de Panos Koutras, 2009), une version queer du mythe d'EEdipe », Transtext(e)s Transcultures 跨文本跨文化 [En ligne], 8 | 2013, mis en ligne le 02 décembre 2013, consulté le 19 avril 2019. URL : http://journals.openedition.org/transtexts/487 ; DOI : 10.4000/ transtexts. 487 


\section{Transtext(e)s Transcultures 跨文本跨 文化}

Journal of Global Cultural Studies

$8 \mid 2013$ :

Genre et filiation : pratiques et représentations

Récits de filiati’ $n$ et (ré)écriture de s’ $i$

\section{Strella (film de Panos Koutras, 2009), une version queer du mythe d'OEdipe}

Strella (A woman's way, Panos Koutras, 2009): a queer version of the CEdipus myth

\section{SOPHIE COAVOUX}

\section{Résumés}

Français English

Troisième long métrage du cinéaste grec Panos Koutras, Strella ( $A$ woman's way), est sorti en 2009. Entièrement autoproduit, le film a connu à sa sortie un succès international, notamment au Festival du film de Berlin où il a été sélectionné dans la section Panorama. Strella est généralement classé par la critique dans la mouvance queer du cinéma et s'inscrit également dans une tendance nouvelle du cinéma grec, puisque Koutras fait partie du collectif FoG (Filmmakers of Greece). Le sujet, comme la forme du film, est audacieux, violent, volontairement dérangeant, à la limite de l'indicible. Il s'agit d'une immersion dans l'univers athénien du transsexualisme, où se mêlent des êtres blessés, marginaux, hors norme - prostitués, anciens détenus, toxicomanes -, univers où sont bousculées les normes officielles de l'identité et du désir. Mais ce n'est pas, à proprement parler, un film sur le transsexualisme puisque le thème majeur de Strella est celui de la filiation. On peut alors considérer le film comme une relecture queer, et plus, une réécriture, très libre, des fondements de la culture grecque, de l'antiquité à la culture populaire : le mythe d'Edipe et la tragédie de Sophocle, Edipe roi, mais aussi la Callas, Mélina Mercouri. Cet article vise à analyser ce récit de filiation queer et à montrer en quoi Strella est une tentative de renouveler la réflexion sur la famille autant que celle sur l'identité sexuelle et genrée, en défaisant les normes culturelles imposées au genre, à la filiation et à l'amour. 
Third feature film of the Greek filmmaker Panos Koutras, Strella (A woman's way), released in 2009, was an international success. It was selected at the Cinema Festival in Berlin in the Panorama section. Critics generally identify Strella as an example of queer cinema but it is also part of a new trend in contemporary Greek cinema, Koutras being a "foggist" (a member of FoG, Filmmakers of Greece). The subject, like the form of the film, is bold, violent, deliberately disturbing, almost unspeakable. It is an immersion in the Athenian world of transsexualism, where are living side-by-side wounded people, the fringes of the society, misfits - prostitutes, (ex-)prisoners, drug addicts - a world where the official standards of identity and desire are disrupted. But this is not, strictly speaking, a film about transsexualism, as the major theme is kinship. Then we can consider the film as a queer revision, and more, a queer rewriting, of the foundations of Greek culture, from Antiquity to popular culture : the myth of CEdipus and Sophocles' tragedy (Edipus the King), but also Maria Callas and Melina Mercouri. This paper aims to analyse this narrative of queer kinship, to show how Strella is an attempt to renew traditional understandings of the family and sexual, gendered identities, and to dismantle the cultural norms imposed on gender, kinship and love.

\section{Texte intégral}

$1 \quad$ Troisième long métrage du cinéaste grec Panos Koutras, Strella ( $A$ woman's $w a y)^{1}$, est sorti en 2009. Entièrement autoproduit, le film a aussitôt connu un succès international, notamment au Festival du film de Berlin où il avait été sélectionné dans la section Panorama. En Grèce également, pays très conservateur encore régi par le dogme " patrie, religion, famille », le film a contre toute attente été bien accueilli tant par le public que par la critique, loin du scandale que certains attendaient.

$2 \quad$ Strella est généralement classé par la critique dans la mouvance queer du cinéma $^{2}$ et s'inscrit également dans une tendance nouvelle du cinéma grec, puisque Koutras fait partie du collectif FoG (Filmmakers of Greece) ${ }^{3}$. Né d'une véritable mutinerie dans le paysage cinématographique grec, ce collectif réunit de jeunes réalisateurs désireux d'adopter une posture de rupture par rapport à la tradition du cinéma grec et soucieux de rendre compte des profondes mutations de la société du pays, en montrant une Grèce plurielle, hétérogène, discontinue.

3 Le sujet, comme la forme du film, est audacieux, violent, volontairement dérangeant, à la limite de l'indicible. Il s'agit d'une immersion dans l'univers athénien du transsexualisme, où se côtoient des êtres blessés, marginaux, hors norme - prostitués, anciens détenus, toxicomanes -, où sont bousculées les normes officielles de l'identité et du désir. Mais ce n'est pas, à proprement parler, un film sur le transsexualisme puisque le thème majeur de Strella est celui de la filiation, sujet qui affleurait déjà dans les deux précédents longs métrages de Koutras, L'Attaque de la moussaka géante (1999) et Alithini Zoï (La vraie vie, 2004). À cet égard l'œuvre se trouve précisément à la croisée des questions du genre et de la filiation. Selon Koutras, c'est le traitement - radical - du thème de la filiation qui lui a probablement valu la frilosité des producteurs grecs. Tous ont en effet refusé de soutenir ce film absolument transgressif qui s'articule autour d'une problématique œdipienne, en abordant de manière frontale le tabou de l'inceste (thème qui apparaissait déjà dans Alithini Zoï, La vraie vie). En ce sens, on peut considérer Strella comme une relecture queer, et plus, une réécriture, très libre, des fondements de la culture grecque, de l'antiquité à la culture populaire : le mythe d'Edipe et la tragédie de Sophocle, Edipe roi, mais aussi les figures de la Callas et de Mélina Mercouri. Pour Judith Butler, Strella est sans doute la plus importante 
contribution culturelle récente au regard œedipien sur la parenté queer et participe également d'une réflexion plus large sur la sexualité et la parenté :

I think that Strella is perhaps the most important cultural contribution in recent years to thinking about oedipalization within queer kinship, as well as about contemporary challenges to understandings of sexuality and kinship, all through a meditation on very contemporary modes of living and loving that nevertheless draw on ancient norms. ${ }^{4}$

Nous nous proposons d'analyser ce récit de filiation queer et de montrer en quoi Strella est une tentative de renouveler la réflexion tant sur la famille que sur l'identité sexuelle et genrée.

5 Giorgos, un quinquagénaire énigmatique, sort de prison après avoir purgé une peine de quinze ans. Dès sa sortie, il se met en quête de son fils, dont il semble avoir perdu la trace depuis son incarcération. Il élit domicile dans un hôtel minable où il est abordé par Strella, un transsexuel qui se prostitue et chante dans un cabaret trans du centre d'Athènes, Koukles (Poupées). Cette rencontre, qui semble n'être au départ qu'une simple passe, évolue très vite en une relation amoureuse et le film prend alors des airs de love story un peu kitsch, comme le concède Koutras. ${ }^{5}$ Mais tant Giorgos que Strella, qui demeurent plus que laconiques sur leur passé respectif, portent chacun en eux des blessures ouvertes et des secrets épouvantables. Leur vérité finit par éclater, à travers une avalanche de révélations insupportables : Strella est en fait Léonidas, le fils que Giorgos recherchait, et les amants se révèlent donc père et fils. Leur rencontre n'avait rien de fortuit, mais avait été provoquée par Strella qui, alors qu'elle voulait seulement revoir son père, se trouve à l'origine de cette transgression involontaire. Le crime de Giorgos nous est également révélé : témoin de la relation incestueuse entre son fils Léonidas et l'oncle maternel de ce dernier, il a assassiné celui-ci, avant d'être jugé pour homicide puis incarcéré. Le drame prend alors le relais de la love story et le film se transforme dès lors en véritable tragédie grecque, écho de mythes primordiaux et de tabous : Edipe, l'inceste, le crime, la vengeance. Mais ultime coup de théâtre : cet horizon d'attente tragique est démenti par un véritable happy end, qui dévie le film du champ de la littérature oraculaire dans lequel il semblait initialement s'inscrire. ${ }^{6}$

$6 \quad$ Ces multiples rebondissements de l'intrigue et ces ruptures invraisemblables rendent compte de la volonté affichée du réalisateur d'échapper à tout cliché narratif et normatif, en inscrivant son film dans une esthétique queer. Il nous semble impératif de décortiquer au préalable le mécanisme du film pour pouvoir comprendre ensuite le traitement de la question de la filiation.

$7 \quad$ Strella est en effet une véritable entreprise de déconstruction, et de bricolage, tant sur le fond que sur la forme. Dans ses films précédents déjà, et surtout avec L'Attaque de la moussaka géante, Koutras avait mis en œuvre sa prédilection pour le mélange des genres (série $Z$ loufoque, filiation avec Ed Wood et surtout, hommage à John Watters et Divine). Avec Strella, il joue encore davantage sur les combinatoires, mélange les registres et les styles, le film échappant ainsi à toute catégorie : soap opéra, cinéma de genre, comédie, comédie romantique, tragédie, mélodrame. À ce propos, un critique a très justement décrit Strella comme " Un film grec qui débute comme du Melville, continue comme du Fassbinder et se termine comme du Disney ». ${ }^{7}$ Ce parti pris esthétique sert avant tout un certain usage de l'ironie, comme mise à distance, qui vise à déplacer le regard du spectateur. En cela, le film relève 
parfaitement de l'esthétique camp, parfaitement analysé par Susan Sontag dans ses Notes on Camp $(1964)^{8}$, qui repose « à la fois sur le travestissement, la parodie, la théâtralité, la pose et l'artificialité $»{ }^{9}$ La mise à distance passe essentiellement par un jeu de transposition doublé d'une subversion des références. Dans ce film placé sous le signe du pastiche, le champ référentiel est éclectique et Koutras procède par citations, selon une technique de collage, assemblant çà et là des fragments de mythes, de tragédie et de culture populaire. Si le film a vocation à porter un message universel, il n'en comporte pas moins nombre de références culturelles grecques, dont la juxtaposition fait partie intégrante de l'entreprise de déconstruction qu'il constitue, pensé, selon Koutras, comme une " remise en cause des stéréotypes et des idées reçues en Grèce » et un " combat contre les préjugés et ce qui représente, [selon Koutras], un "poids" dans [l'] héritage culturel [grec] ». Le réalisateur déclare en effet dans une interview :

Nous avons longtemps été obligés de porter les valeurs de la Grèce
Antique et en proposer une relecture m'a toujours stimulé. En inscrivant
les racines de notre culture dans le monde contemporain, je tenais à
montrer que l'on peut et que l'on doit faire évoluer un héritage comme
celui-là. La Grèce est un pays moderne, même s'il est plus rigide,
conservateur et bourgeois que le reste de l'Europe parce qu'il fait le
grand écart entre Orient et Occident. Dans la Grèce antique,
l'homosexualité n'existait pas, il n'y avait pas de traîtres mais que des
héros, l'esclavage n'était qu'un détail : une société idéale, en somme
(rires)... Aujourd'hui, encore davantage que par le passé, il existe une
autre Grèce : celle des gays, des transsexuels, des pauvres, des marginaux
et cette Grèce-là véhicule une vérité indiscutable. Le peuple aussi s'est
approprié des icônes comme La Callas ou Mélina Mercouri et c'est lui qui
est porteur de changements sociaux. Chez les transsexuels notamment, il
y a une répercussion plutôt juste de ces valeurs et je voulais décrire leur
quotidien avec le maximum d'authenticité.10

La première des références, du moins la plus évidente, est empruntée à la culture populaire. Strella, à la fois nom du personnage et titre du film, est un néologisme, forgé de la contraction du prénom grec « Stella » et du substantif " $\boldsymbol{\tau} \boldsymbol{\rho} \dot{\boldsymbol{\lambda} \lambda \boldsymbol{\alpha}}$ », qui signifie " folie ». Mais pour le spectateur grec, ce nom et ce titre font immédiatement écho au film Stella, réalisé en 1955 par Michel Cacoyiannis, qui révéla au public Mélina Mercouri comme l'incarnation parfaite de la femme libre. Le mélodrame de Cacoyiannis (au sens cinématographique du terme, dans le contexte du cinéma néo-réaliste des années 1950) met en scène une chanteuse de cabaret que sa passion amoureuse et sa passion pour la liberté précipiteront vers une issue fatale. Construit comme une tragédie grecque, le film bouleversa la représentation de la femme dans le cinéma grec ${ }^{11}$, et Mélina Mercouri interpréta par la suite d'autres rôles illustres qui firent d'elle une véritable icône de la femme libre (Jamais le dimanche, Jules Dassin 1959). Ce parallèle évident entre Stella et Strella se trouve d'ailleurs renforcé par d'autres références, plus indirectes, au film de Cacoyiannis ou à Mélina Mercouri. ${ }^{12}$

$9 \quad$ Venons-en à présent aux références antiques. Fait significatif, l'action du film se déroule principalement à Athènes, mais loin de la carte postale et du symbole prestigieux que représente la capitale grecque, Koutras a préféré montrer l'urbanisation agressive de cette métropole moderne, hostile, particulièrement pour des êtres fragiles ${ }^{13}$ : quartiers populaires, scènes de rues, 
voie ferrée, prison, poubelles, quartiers en déshérence, où l'on entrevoit les ombres des laissés-pour-compte. Le Parthénon - que, par parenthèse, Koutras fait brûler dans Alithini Zö - n'apparaît que furtivement, à travers la fenêtre d'un hôtel ou, démythifié, dessiné sur un sac, camelote pour touristes, avec le slogan ironique « Live your myth " (slogan d'une campagne de promotion de l'Office national du tourisme).

Au-delà de ce cadre symbolique, la principale référence antique que l'on relève, implicite celle-là, apparaît évidemment à travers la réactualisation du mythe d'Edipe et de l'histoire des Labdacides. Précisons bien que c'est le mythe d'Edipe qui est ici convoqué et non le complexe d'Edipe, tel qu'il a été développé par Freud. Loin de la théorie psychanalytique, Koutras transpose, transforme puis détourne l'Edipe roi de Sophocle. Strella est bien sûr Edipe. D'ailleurs, comme lui, elle évoque une blessure au pied (Edipe, nom qui signifie littéralement " celui qui a les pieds enflés »). Mais à la différence d'Edipe qui commet l'inceste avec sa père, c'est avec son père, Giorgos alias Laïos, que Strella transgresse le tabou suprême. À noter que Giorgos, tout comme Laïos, se caractérise par son homosexualité (au début du film, on comprend qu'il est l'amant de son compagnon de cellule ${ }^{14}$ ). Jocaste, la mère d'Edipe, a disparu dans le récit de Koutras, d'où les femmes sont d'ailleurs totalement absentes. Cette absence est vraisemblablement à comprendre comme un clin d'œil à la tragédie antique où tous les rôles, même féminins, étaient incarnés par des hommes. En revanche, le devin Tirésias réapparait, sous les traits de Mairi, une transsexuelle en phase terminale d'un cancer - ce Tirésias queer renvoie à certaines traditions du mythe selon lesquelles le personnage se situait à l'intersection des deux sexes. Figure maternelle de substitution pour Strella, lorsqu'elle apprend la nature des liens entre cette dernière et son père, elle la prévient qu'elle est coupable d'hybris - c'est le terme qu'elle emploie en se référant à l'Antiquité. Elle l'avertit de ce fait que son acte les expose, elle et son père, à un châtiment inéluctable (la némésis). Tout comme lorsque, dans la tragédie de Sophocle, Tirésias révèle à Edipe sa double faute (parricide et inceste) et l'avertit qu'il court à sa perte, Mairi déclare à Strella :

« C'est un tabou. Tu comprends ? Ces choses ne se font pas. »

11 Pourtant, le dénouement choisi par Koutras échappe au genre tragique : il n’y aura ni intervention divine (puisque, comme le dit Strella « La déesse des trans est partie en vacances, ou elle est en prison »), ni manifestation de la némésis (mais il y aura malgré tout catharsis). Et c'est en cela que réside l'originalité du film qui se distingue donc de la tragédie grecque et du mélodrame grec des années 1950 qui s'en inspirait. ${ }^{15}$ Ainsi la référence explicite à Sophocle, à Stella ou à d'autres films comme Evdokia d'Alexis Damianos (1971), met en exergue cette différence essentielle : Stella et Evdokia, comme Edipe, et contrairement à Strella, sont punis pour avoir transgressé les règles. Koutras n'a pas voulu adopter cette perspective fataliste, en refusant de montrer Strella expiant sa faute par la mort. ${ }^{16}$

12 Dans ce contexte, qu'en est-il du traitement du thème de la filiation, et plus particulièrement de la question centrale des rapports père/fils entre Giorgos et Strella ? Nous évoquions plus haut la transgression involontaire de l'inceste. En effet, l'histoire d'amour entre Strella et Giorgos, telle qu'elle se dessine dans le film, n'est pas le fruit d'une passion aveugle. Avant même leur rencontre, 
Strella connait parfaitement l'identité de Giorgos et le lien biologique qui les unit. En revanche, elle n'a pas prémédité l'inceste et tente au départ d'esquiver les avances de son père. Si elle se garde par la suite, volontairement, de révéler ce secret inavouable, ce n'est pas par vengeance, comme le supposent initialement Giorgos et Mairi, mais motivée par l'angoisse de la perte, portée par le besoin, absolu, de ne plus perdre, comme par le passé, cet homme qui lui a manqué. Et c'est ainsi qu'elle se justifie, face à la fureur de son père :

\section{Je voulais seulement te voir. Je ne voulais pas que ça se passe comme ça. J'avais besoin de toi. [...] Je savais que tu étais mon père mais je ne te ressentais pas comme tel [...]. Tu étais comme un étranger. [...] En réalité, nous sommes deux étrangers. [...] Ne pars pas. On va trouver une solution.}

En définitive, ce n'est pas la transgression du tabou de l'inceste qui confère au film son caractère subversif mais la solution queer qu'il propose à cette situation intenable. Une fois la vérité révélée, Giorgos devra en effet affronter les vieux démons de son enfance manquée, pour accepter son besoin de lien affectif, son besoin de l'autre, et provoquer la réconciliation. Il exprime cela dans une phrase choc à propos de la paternité :

Finalement je suis un homme heureux. Tu m'as permis de t'aimer de toutes les manières qu'un père peut aimer son enfant. [...] Je ne veux pas te perdre.

Précisant dans une interview que Giorgos est simplement un père et non le porte-drapeau de la condition paternelle, Koutras ajoute :

Je pense que c'est cette phrase-là qui a coupé le film de toute subvention, mais je continue de la revendiquer parce que je n'ai pas réalisé une œuvre didactique : ces propos appartiennent au personnage, c'est SON avis, SA façon de résoudre une situation intenable. ${ }^{17}$

D’ailleurs, le dénouement heureux du film ne dit pas ce qui va se passer ensuite entre Strella et son père, Koutras ayant voulu « que le devenir de leur relation reste en pointillés à la fin du film : chaque spectateur a le droit d'imaginer le rôle qu'ils vont tous deux jouer au sein de leur nouvelle famille. ${ }^{18}$ En effet, selon lui « un film doit suggérer des pistes de réflexions, pas imposer une vérité. ${ }^{19}$ Strella se présente ainsi comme une question ouverte, et, mêlant imbroglio œedipien, subversion du genre et désir homosexuel, tout en échappant aux stéréotypes, il invite à s'interroger sur la normalité, sur ce qui est permis, sur les limites - celles du désir, de la sexualité, des rapports humains, de la filiation. L'idée d'une conception universelle et atemporelle de la filiation et des relations filiation/genre se trouve ainsi remise en question par la confrontation à l'altérité. Aussi le paradigme traditionnel de la filiation laisse-t-il place à un ordre symbolique post-œdipien ${ }^{20}$ où deviennent possibles des modes de vie qui échappent aux structures normatives de la famille traditionnelle. Koutras montre ainsi les failles et les limites de la filiation de sang, et privilégie la filiation de cœur. Il revendique ainsi sa croyance en une vision large de la famille qui englobe aussi bien les parents qu'un voisin ou des amis » (puisque selon lui « seul compte le besoin et le bonheur de l'autre ${ }^{21}$, à une époque où " la notion de famille au sens traditionnel ne rend plus compte de la réalité ».

En effet, pour Strella, « La famille, [c'est] la malédiction des trans. ». Tous 
les personnages du film sont seuls, privés de liens familiaux, de par leur marginalité, leur mode de vie (prostitution, transsexualité, prison) et souffrent de ce manque. Autour de Strella (qui, comble du paradoxe, est loin de la folie mais incarne au contraire la raison et les qualités du cœur et fait preuve de bon sens et de générosité) se nouent des liens très forts d'affection et de solidarité, comme au sein d'une nouvelle famille. Elle rend de menus services à Mairi et l'accompagne dans son combat contre le cancer, et se chargera même de la préparer, selon ses dernières volontés, pour ses funérailles. En retour, cette figure maternelle drag queen, lui prodigue, avec l'attention et la tendresse d'une véritable mère, tous les conseils nécessaires. Strella s'occupe également d'Alex, un jeune homosexuel un peu perdu dont elle accueillera même sous son toit la petite sœur d'Alex, Bou, après le décès de leur mère toxicomane. De manière générale, tous les personnages tissent entre eux des liens très forts d'amour et de solidarité, comme pour pallier le manque de structure familiale. Cette image de famille recomposée, bricolée, est l'un des messages forts du film, qui selon Koutras, n'est pas « une douce utopie », mais une réalité :

$\mathrm{Au}$ contraire, c'est une réalité, une aspiration moderne qui dépasse le cadre du film. Je l'ai constatée chez tous ces gens qui vivent en marge de la société et qui ne bénéficient plus de sa protection : ils sont obligés de se rapprocher et de se soutenir face à un monde très dur, violent. ${ }^{22}$

Dans le film, le genre, la famille et les rapports de filiation sont successivement déconstruits puis bricolés, au même titre que Strella passe son temps au bricolage (symbolique) d'objets cassés ou récupérés dans une poubelle (une télévision, un lustre, une lampe). Koutras tenait en effet à illustrer l'idée selon laquelle chacun peut contrôler son identitée ${ }^{3}$, et de fait chaque personnage contrôle, bricole et construit/ou reconstruit sa propre identité au cours de la narration qui s'inscrit résolument dans une optique constructiviste. À cet égard, la question du nom, centrale dans Strella, est particulièrement parlante. Le nom de l'hérö̈ne est lui-même un bricolage, l'avatar d'un nom féminin qu'elle s'est choisi après avoir renoncé à son identité et à son prénom de garçon (Léonidas), et à son tour détourné par ses amis. Elle refuse donc celui que ses parents lui avaient donné et s'enchoisit un nouveau, dans un acte symbolique de création de soi. Dans le même ordre d'idée, lorsque Mairi se réfère aux tragiques grecs pour expliquer à Strella en quoi elle est coupable d'hybris, elle féminise les noms de Sophocle et d'Euripide. Bien loin d'être anodins, ces " détails » renvoient à la dimension performative de réappropriation du «naming » et du " self naming », développée par Judith Butler, face au pouvoir normatif du langage et au processus de « dépossession »/ « réappropriation » d’identité qu'il induit :

[...] the act of naming can be a form of appropriation, and we see this, for instance, in the Bible, when God extends his dominion precisely through naming everything in sight (we might say that what he names comes into sight for the first time). So naming can operate in the service of a sovereign form of the performative. For us, the question will be, what form can and does naming take when it seeks to undo the sovereign status of the one who names ? One way of starting the inquiry, then, would be to take seriously the fact that the one who names is always also named. In other words, whoever uses language to name is already interpellated into a language, even used by language prior to any deliberative use of language. This would constitute a general condition of the name that precedes and exceeds any particular instance of naming, 
including self-naming. Of course, self-naming is important, and we surely see this, for instance, when transgendered people struggle with what to name themselves, how to change the name, how to petition that others use the name that they wish. In such instances we can see how the problem of desire suffuses the issue of the name. [...] So perhaps what appear to us, you and me, as a possible tension between particularism and universalism is actually rethinkable in light of a general politics of naming. If we are always named by others, then the name signifies a certain dispossession from the start. I $\mathrm{f}$ we seek to name ourselves, it is still within a language that we ever made. And if we ask to be called by another name, we are in some ways dependent on those we petition to agree our demand. There seems to be an overdetermination of the social at the site of the name, so however particularistic we want the name to be, it exceeds us and confounds us. At the same time, its generality is the condition of our particularity, the instance of its singular renewal and innovation, sometimes a moment of poiesis : "Strella !". 24

L'épilogue du film, qui prend la forme d'un happy end inattendu, illustre parfaitement la conception de la famille du réalisateur. On assiste à une scène de réveillon de fin d'année qui répond en première analyse à tous les clichés du genre : la maison est décorée, au centre du salon trône un sapin de Noël, et chacun s'affaire à la préparation du repas, « dans la joie et la bonne humeur ». La liste des invités est quant à elle beaucoup moins classique : autour de Strella, on retrouve Giorgos, son père, Alex le colocataire et sa petite sœur Bou, ainsi que l'ancien amant et compagnon de cellule de Giorgos, qui a lui-même invité un ami Youri, un ancien détenu, pour éviter qu'il reste seul. Entre tous les membres de cette famille queer règne une harmonie sans faille. Il faut souligner en outre que Koutras n'a pas cherché ici à faire un pastiche de la famille en assignant à chacun un rôle et une identité prédéfinis qui réponde à un ordre familial hiérarchisé : au contraire, les relations entre les personnages sont uniquement fondées sur le respect mutuel et l'amour. La dimension ironique de cette dernière scène ne fait évidemment aucun doute. Mais ce qui constitue sans doute l'élément le plus subversif c'est que cet épilogue pied-de-nez laisse in fine l'impression que ce « nouvel ordre symbolique » qui régit les rapports entre les individus, ce nouveau type de famille, issu ici d'un monde marginal et interlope, est supérieur en humanité au modèle traditionnel, impression que véhicule d'ailleurs l'ensemble du film.

S'il ne se veut ni didactique ni pamphlétaire, et s'il ne peut être assimilé à un conte moral, Strella n'en pose pas moins un regard critique sur la société grecque contemporaine. Méditation sur les normes culturelles imposées au genre, au désir, à l'amour, et sur la conception traditionnelle des liens de filiation, le film vise avant tout à bousculer le spectateur et à le pousser à s'interroger. ${ }^{25}$ En traitant le thème rare et violent de l'inceste, sur fond d'esthétique queer, Koutras semble adhérer à une philosophie camp, " philosophie de la subversion et du doute, qui questionne la légitimité et le sérieux revendiqué des hiérarchies sociales et culturelles $» .^{26}$ Sans jamais sombrer dans le sordide, et en alliant la crudité du propos (et parfois des images) à une infinie pudeur portée par des personnages magnifiques, Koutras a réussi, je crois, à réaliser un film profondément humain.

20 Par ailleurs, il s'inscrit avec Strella dans une tendance du nouveau cinéma grec indépendant (cf. collectif FoG), qui suscite depuis plusieurs années un intérêt international. Signalons simplement pour finir que l'un des thèmes de prédilection de ce nouveau cinéma est celui de la famille. ${ }^{27}$ Depuis les années 
2000 en effet, plusieurs longs métrages grecs mettent en effet à mal la représentation traditionnelle de la famille grecque, véritable institution, en soulignant tout ce qu'elle recèle en elle de noirceur : Canine, de Giorgos Lanthimos, qui reçut le Prix « Un certain regard » à Cannes en 2009, Alps, du même réalisateur, sélectionné en compétition à la Mostra de Venise en 2011, où il reçut le prix du meilleur scénario, ou plus récemment, Miss Violence, d'Alexandros Avranas, doublement récompensé au dernier festival de Venise (Lion d'argent meilleur réalisateur, coupe Volpi meilleure interprétation masculine).

\section{Notes}

1 Strella, Panos Koutras, Feelgood entertainment.

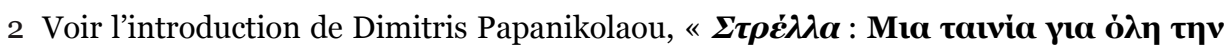
oukoyéveıа ", Strella, Panos Koutras, Panayiotis Evaggelidis, éditions Polychromos Planitis, Athènes, 2010, pp. 9-24. Sur le cinéma queer en Grèce, voir l'article de Georges

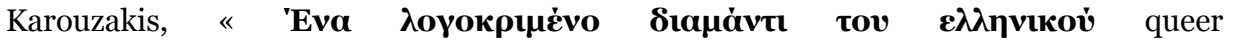

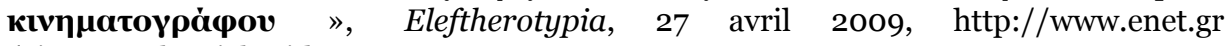
$/$ ? $\mathrm{i}=$ news.el.article\&id $=38598$.

3 www.fogfilms.org

4 Judith Butler, conversations with Athena Athanassiou, Dispossession : The Performative in the Political, Cambridge, Polity Press, 2013, p. 59.

5 Cet entretien a eu lieu le 31 octobre 2009, à Montpellier, lors du $31^{\mathrm{e}}$ festival international du cinéma méditerranéen. Transcription et présentation de Christiane Passevant (« Histoire de famille. Entretien avec Panos H. Koutras ») http://chroniquesrebelles.info/spip.php?article538.

6 Contrairement à l'interprétation de Jacques Mandelbaum pour qui le film est « fidèle à la littérature oraculaire ». Voir « Strella : une version 'queer' de la culture antique », Le Monde, 17.11.2009.

7 Serge Kaganski, « Strella », Les Inrockuptibles, 16 novembre 2009, www.lesinrocks.com/cinema.films-a-l-affiche/strella.

8 Dans Against Interpretation and Other Essays, Pinguins Classics, 2009 (rééd.).

9 Florence Tamagne, Mauvais genre. Une histoire des représentations de l'homosexualité, Paris, La Martinière, 2001, p. 251. Sur le camp, voir aussi Umberto Eco, Histoire d la laideur, Paris, Flammarion, 2007, pp. 408-419.

10 Interview de Panos Koutras, "Strella n'est pas un film militant ou politique », Universciné, 2009, https://www.google.com/url?q=http://www.universcine.com /articles/panos-koutras-strella-n-est-pas-un-film-militant-ou-politique\& $\mathrm{sa}=\mathrm{U} \& \mathrm{ei}=\mathrm{C}_{1} \mathrm{M}_{4} \mathrm{U}$ oGjNdCS7AajwYDICQ\&ved=oCBcQFjAG\&client=internal-uds-cse\& usg=AFQjCNFtZ4OGFj-8gojb5ZgQEKHNaAHjjg.

11 Voir Stéphane Sawas, «Image(s) de femme(s) dans Stella de Michalis Cacoyannis et Et Dieu créa la femme de Roger Vadim », Paris, Desmos, nº12, 2003, pp. 50-59.

12 Strella, comme Stella, est un personnage fantasque, extraverti et joyeux, malgré les blessures de la vie, avides de liberté. Toutes deux sont chanteuses de cabaret. Dans le film de Koutras, une scène en particulier, tournée dans le cabaret Koukles, montre une performance réalisée par un transsexuel incarnant Mélina Mercouri et reprenant la

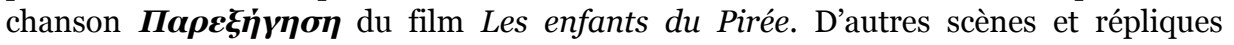
opèrent ce jeu d'intertextualité avec d'autres films, en référence à Mélina Mercouri.

13 Interview de Panos Koutras, «Strella n'est pas un film militant ou politique », ibid.

14 Mais il ne s'agit pas de Chrysippe : ils sont liés dans une relation amoureuse sincère qui ne se rapproche en rien de la relation entre Laïos et Chrysippe (qui entraînera le suicide de ce dernier, après le viol de Laïos).

15 Sur le sujet, voir Le cinéma grec, sous la direction de Michel Démopoulos, Paris, Cinéma/Pluriel, Éditions du Centre Georges Pompidou, 1995, p. 107 (in Niki 
Karakitsou-Dougé, « Le mélodrame grec : "une esthétique de l'étonnement" », pp. 107-112).

16 Entretien (Christiane Passevant), ibid.

17 Interview de Panos Koutras, «Strella n'est pas un film militant ou politique », ibid.

18 Idem.

19 Idem.

20 Sur ce point, voir Butler-Athanassiou, chapitre 5 " (Trans)possessions, or bodies beyond themselves », en particulier p. 57 : «In Strella, queer genealogy troubles and redefines dominant histories of kinship, nation, memory, desire and sexual alliance. The riveting scene of the revelation of the transgender secret - a scene that plays cinematographically with light and shadows, bodies and specters - de-mythifies and re-mythifies a desire of recognition that lays bare the limits of the representable and the effaceable and defies the elementary structures of kinship intelligibility. In the scene of Strella's coming-out as transgendered to her lover/father, the Law of the Father is displaced just as the two lovers (male father and transgender child) are emerging in a post-Oedipal symbolic order, beyond genealogical inheritance and disinheritance, beyond possession and dispossession ». Voir aussi p. $58 \mathrm{sqq.}$

21 Interview de Panos Koutras, «Strella n'est pas un film militant ou politique », ibid.

22 Interview de Panos Koutras, «Strella n'est pas un film militant ou politique », ibid.

23 Idem. : " Par exemple, à travers le personnage de Mary qui est atteinte d'un cancer, j'ai voulu montrer que l'on pouvait contrôler son identité jusqu'à la mort : elle organise héroïquement ses funérailles comme si elle pouvait vaincre l'inéluctable. ».

24 Butler-Athanassiou, pp. 137-138 (dans le chapitre 12 " Dispossessed languages, or singularities named and renamed »). Sur le sujet, voir aussi Judith Butler, Bodies that matter. On the discursive limits of "sex", New York, Routeldge, 1993, p. 227.

25 Sur les liens entre genre et filiation, voir l'article de Judith Butler, « Is Kinship Always Already Heterosexual ? ", Differences: A Journal of Feminist Cultural Studies, Duke University Press, Volume 13, Number 1, Spring 2002, pp. 14-44, http://muse.jhu.edu/journals/dif/summary/v013/13.1butler.html.

26 Mauvais genre, p. 251.

27 Sur le traitement du thème de la famille dans le cinéma grec contemporain, voir

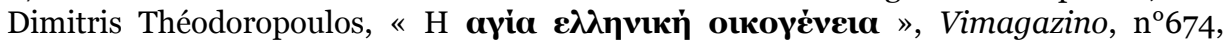
15.09.2013, p. 16.

\section{Pour citer cet article}

Référence électronique

S` phie COAVOUX, « Strella (film de Pan`s K` utras, 2009), une versi` n queer du mythe d'OEdipe », Transtext(e)s Transcultures 跨文本跨文化 [En ligne], 8 | 2013, mis en ligne le 02 décembre 2013, c’ nsulté le 13 janvier 2014. URL :

http://transtexts.revues.' $\mathrm{rg} / 487$

\section{Auteur}

\section{Sophie COAVOUX}

$S^{\prime}$ phie COAVOUX est Maître de c' nférences de grec m`derne à l'Université Jean M` ulin (Ly` $n$ ) et membre de l'IETT (Institut d'Études Transtextuelles et Transculturelles). Sa thèse de $d^{\prime} \mathrm{ct}^{\prime}$ rat sur le $\mathrm{p}^{\prime}$ ète grec $C$. P. Cavafy a été $s^{`}$ utenue en 2008. Ses travaux $p^{`}$ rtent sur la littérature grecque $m^{`}$ derne et sur les questi ns du genre et de la diasp' ra.

$S^{\prime}$ phie COAVOUX is Maître de c' nférences in M` dern Greek Studies at the Jean $M{ }^{\prime}$ ulin University (Ly` $n$ 3) and a member ' $f$ the IETT (Institute f' $r$ Transtextual and Transcultural Studies). Her d` ct ' rate ' $n$ the Greek $p$ ' et C. P. Cavafy was c' mpleted in 2008. Her current research $f$ ' cuses ' $n$ M’ dern Greek literature, gender and diasp' ra.

Articles du même auteur 
Editorial [Texte intégral]

Paru dans Transtext(e)s Transcultures 跨文本跨文化, 8 | 2013

Greek Diaspora and Hybrid Identities: Transnational and Transgender

Perspectives in Two novels: Loaded, by Christos Tsiolkas (Australia) and

Middlesex, by Jeffrey Eugenides (USA) [Texte intégral]

Paru dans Transtext(e)s Transcultures 跨文本跨文化, 7 | 2012

Aspects de l'hellénisme dans l'œuvre poétique de Constantin Cavafy [Texte intégral]

Paru dans Transtext(e)s Transcultures 跨文本跨文化, 1 | 2006

\section{Droits d'auteur}

(C) Tous droits réservés 